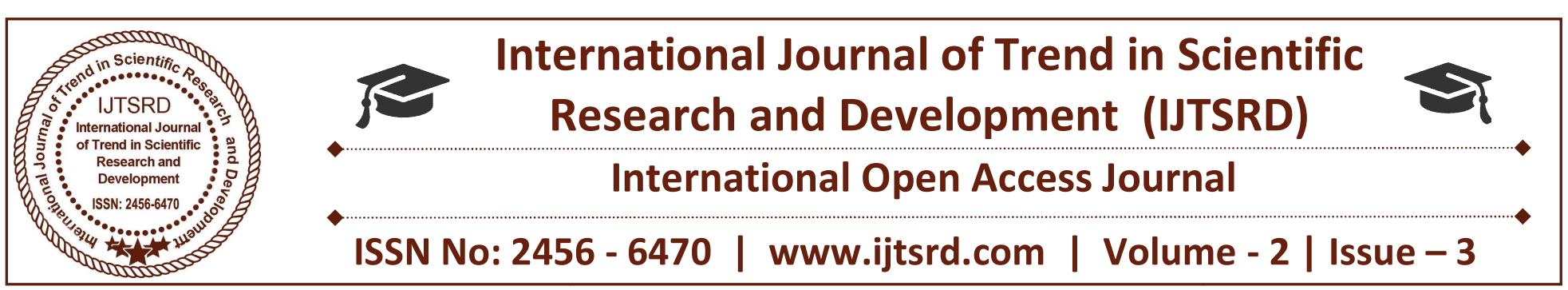

\title{
DCT Based Robust \& Secured Digital Image to Audio Watermarking Algorithm
}

\author{
Ikchha Pandey \\ Research Scholar, Department of \\ Electronics \& Communication, \\ Gyan Ganga College of \\ Technology, Jabalpur, \\ Madhya Pradesh, India
}

\author{
Siddarth Bhalerao \\ Assistant Professor, Department of \\ Electronics \& Communication, \\ Gyan Ganga College of \\ Technology, Jabalpur, \\ Madhya Pradesh, India
}

\author{
Papiya Dutta \\ Associate Professor \& H.O.D., \\ Department of Electronics \& \\ Communication, Gyan Ganga \\ College Of Technology, Jabalpur, \\ Madhya Pradesh, India
}

\section{ABSTRACT}

In this paper, a new watermarking based on Discrete Cosine Transform Sub-band coding scheme is presented. This method uses the time domain signal $\&$ process it in frequency domain, while time domain features of the carrier remains same, so no one can identify the hidden data into it. The algorithm is based on Psychoacoustic Auditory Model \& Spread Spectrum theory. It generates a watermark signal using spread spectrum theory \& embeds it into the signal by measuring the masking threshold. Since the watermark is shaped to lie below the masking threshold, the difference between the original \& the watermarked copy is imperceptible. Recovery of the watermark is performed without the knowledge of the original signal. In this work an image logo is taken which is of size $284 \times 177$ as data object called original image, \& an audio of size $158 \mathrm{~KB}$ and duration of $0.7 \mathrm{Sec}$, is taken as carrier object.

Keyword: DCT, Watermarking, Image to Audio watermarking, Password security

\section{INTRODUCTION}

In modern world each and every form of information, like text, images, audio or video, has been digitized. Widespread networks and internet has made it easier and far more convenient to store and access this data over large distances. Although advantageous, this same property threatens the copyright protection.
Media and information in digital form is easier to copy and modify, and distribute with the aid of widespread internet. Every year thousands of sound tracks are released and within a few days are readily available on the internet for download. Without any information on the track itself, it's easy for some one to make profit out of them by modifying the original and selling under a different name. As a measure against such practices and other intellectual property rights, digital watermarking techniques can be used as a proof of the authenticity of the data.

Digital Watermarking is the process of embedding or inserting a digital signal or pattern in the original data, which can be later used to identify the author's work, to authenticate the content and to trace illegal copies of the work.

\section{Requirements}

Some of the requirements of the digital watermarking are:

$>$ The original media should not be severely degraded and the embedded data should be minimally perceptible. *The words hidden, inaudible, imperceptible, and invisible mean that nobody notice the presence of the hidden data.

$>$ The hidden data should be directly embedded into the carrier, rather than into the header of it.

$>$ The watermark should be robust, also it should immune to all types of modifications including channel noise, filtering, re-sampling, cropping, encoding, lossy compressing, digital-to-analog 
(D/A) conversion, and analog-to-digital (A/D) conversion, etc.

$>$ It should be easy for the owner or a proper authority to embed and detect the watermark.

$>$ It should not be necessary to refer to the original signal when extracting a watermark.

\section{Literature Survey}

[1] Digital watermarking has been used for maintaining copyright information of the digital media for a decade. Digital watermarking is a technique used for embedding copyright information in the media files. The media file could be an image, an audio, a video, or a text. Steganography have been used in the Digital watermarking application. Steganography has become more important due to the exponential growth of communication of potential computer information on the internet. Steganography differs from cryptography, such that cryptography hides the contents of secret message, whereas, steganography is about hiding the message in media effectively. This research article gives an overview of steganography, its applications, and difference from cryptography. This research article analyses performance of the Least Significant Bit (LSB), Discrete Cosine Transform (DCT), and Discrete Wavelet Transform (DWT).

[2] Digital multimedia technology has shown a significant progress day to day in various fields such as entertainment, computer vision, security etc. hence, the distribution and use of multimedia data is much easier and faster. In such an open environment, it is convenient to get the access to various information sources. I present time, several issues are arising such as information security, information authentication, ownership or copyright protection with multimedia contents. Therefore, watermarking is breakthrough technique for resolving these issues for digital multimedia content like image, audio and video. I tis paper, to improve the weak points of conventional echo hiding and overcome the problem of less security, we propose an audio watermarking technique based on pseudo-random gray sequences as a confidential key. To satisfy and maximize both imperceptibility and robustness, proposed audio watermarking algorithm based on pseudo-random gray sequences embed multi bit by spreading echo in time domain. The imperceptibility and the robustness of proposed audio watermarking are tested using various attacks and by listening test.

[3] In one's day to day life; internet is growing and became an important part. Digital content can easily be downloaded, copied or edited. Digital content can be secured by various ways. Digital Watermarking is one of the methods for the protection of Digital Content. Digital Watermarking is a method by which data can be secured by hiding data in any image which can work as a carrier image. The carrier image is also known as cover image. Watermarking is an interactive method to protect and identify the digital data. It permits various types of watermarks to be hidden in digital data e.g. image, audio and video. In this work, watermarking has been done using LSB technique, DCT transform and DWT transform. These techniques are compared on the basis of peak signal to noise ratio (PSNR) and normalized correlation (NC). Parameters are compared for various noises like Gaussian noise, Poisson noise, Salt and Pepper noise and Speckle noise.

[4] Authentication and tampering detection of the digital signals is one of the main applications of the digital watermarking. Recently, watermarking algorithms for digital images are developed to not only detect the image tampering, but also to recover the lost content to some extent. In this paper, a new watermarking scheme is introduced to generate digital self-embedding speech signals enjoying the self-recovery feature. For this purpose, the compressed version of the speech signal generated by a speech codec and protected against the tampering by the proper channel coding is embedded into the original speech signal. Experimental results show that the self-embedding speech signal is recoverable with proper speech quality for high tampering rates, without significant loss in the quality of the original speech signal.

[5] Image quality evaluation is very important. In applications involving signal transmission, the Reduced- or No-Reference quality metrics are generally more practical than the Full Reference metrics. In this study, we propose a quality estimation method based on a novel semi-fragile and adaptive watermarking scheme. The proposed scheme uses the embedded watermark to estimate the degradation of cover image under different distortions. The watermarking process is 
implemented in DWT domain of the cover image. The correlated DWT coefficients across the DWT subbands are categorized into Set Partitioning in Hierarchical Trees (SPIHT). Those SPHIT trees are further decomposed into a set of bitplanes. The watermark is embedded into the selected bitplanes of the selected DWT coefficients of the selected tree without causing significant fidelity loss to the cover image. The accuracy of the quality estimation is made to approach that of Full-Reference metrics by referring to an "Ideal Mapping Curve" computed a priori. The experimental results show that the proposed scheme can estimate image quality in terms of PSNR, wPSNR, JND and SSIM with high accuracy under JPEG compression, JPEG2000 compression, Gaussian low-pass filtering and Gaussian noise distortion. The results also show that the proposed scheme has good computational efficiency for practical applications.

\section{Discrete Cosine Transform}

The discrete cosine transform is a technique for converting a signal into elementary frequency components [17]. The DCT can be employed on both one-dimensional and two dimensional signals like audio and image, respectively. The discrete cosine transform is the spectral transformation, which has the properties of Discrete Fourier Transformation [17]. DCT uses only cosine functions of various wave numbers as basic functions and operates on real-valued signals and spectral coefficients. DCT of a 1-dimensional (1-d) sequence and the reconstruction of original signal from its DCT coefficients termed as inverse discrete cosine transform (IDCT) can be computed using equations [17]. In the following, $\operatorname{fdct}(\mathrm{x})$ is original sequence while $\operatorname{Cdct}(\mathrm{u})$ denotes the DCT coefficients of the sequence.

$$
\begin{aligned}
& C_{d u}(u)=\alpha(u) \sum_{x=1}^{N_{v}-1} f_{d u}(x) \cos \left[\frac{\pi(2 x+1) u}{2 N_{u}}\right] \text {, for } u=0,1,2, \ldots, N_{u}-1 \\
& f_{d \tau}(x, y)=\sum_{v=1}^{N_{i-1}} \alpha(u) C_{d \tau t}(u) \cos \left[\frac{\pi(2 x+1) u}{2 N_{1 t}}\right] \text {, for } x=0,1,2, \ldots, N_{\mathrm{lt}}-1 \\
& \text { where } \alpha(u)=\left\{\begin{array}{l}
\sqrt{\frac{1}{N_{1}}} \text { for } u=0 \\
\sqrt{\frac{2}{N_{1}}} \text { for } u \neq 0
\end{array}\right.
\end{aligned}
$$

From the equation for $\operatorname{Cdct}(\mathrm{u})$ it can be inferred that for $\mathrm{u}=0$, the component is the average of the signal also termed as dc coefficient in literature [38]. And all the other transformation coefficients are called as ac coefficients. Some of the important applications of DCT are image compression and signal compression.

The most useful applications of two-dimensional (2d) DCT are the image compression and encryption [17]. The 1-d DCT equations, discussed above, can be used to find the 2-d DCT by considering every row as an individual 1 - $d$ signal. Thus, DCT coefficients of an $\mathrm{M} \times \mathrm{N}$ two dimensional signals $\operatorname{Cdct} 2(\mathrm{u}, \mathrm{v})$ and their reconstruction $f d c t 2(\mathrm{x}, \mathrm{y})$ can be calculated by the equations below:

$$
\begin{aligned}
& C_{d u 2}(u, v)=\alpha(u) \alpha(v) \sum_{z=0}^{M_{21}} \sum_{y=0}^{N_{2 y}} f_{d x 2}(x, y) \cos \left[\frac{\pi(2 x+1) u}{2 M_{2 u}}\right] \cos \left[\frac{\pi(2 y+1) v}{2 N_{2 t}}\right] \\
& f_{d n 2}(x, y)=\sum_{n=0}^{M_{2 u}} \sum_{y=0}^{K_{24}} \alpha(u) \alpha(v) C_{d=2}(u, v) \cos \left[\frac{\pi(2 x+1) u}{2 M_{2 t}}\right] \cos \left[\frac{\pi(2 y+1) v}{2 N_{2 t}}\right]
\end{aligned}
$$

where $u \& x=0,1,2, \ldots, M_{2 t-1}$ and where $v \& y=0,1,2, \ldots, N_{2 t-1}$

$$
\alpha(u)=\left\{\begin{array}{l}
\sqrt{\frac{1}{N_{2 t}}} \text { for } u=0 \\
\sqrt{\frac{2}{N_{2 t}}} \text { for } u \neq 0
\end{array} \quad \& \quad \alpha(v)=\left\{\begin{array}{l}
\sqrt{\frac{1}{N_{2 t}}} \text { for } v=0 \\
\sqrt{\frac{2}{N_{2 t}}} \text { for } v \neq 0
\end{array}\right.\right.
$$

In frequency domain, coefficients are slightly modified. This will make some unnoticeable changes in the whole image and makes it more robust to attack compared to what we have in spatial methods. One of the most popular approaches in this category is the discrete cosines transform (DCT) method.

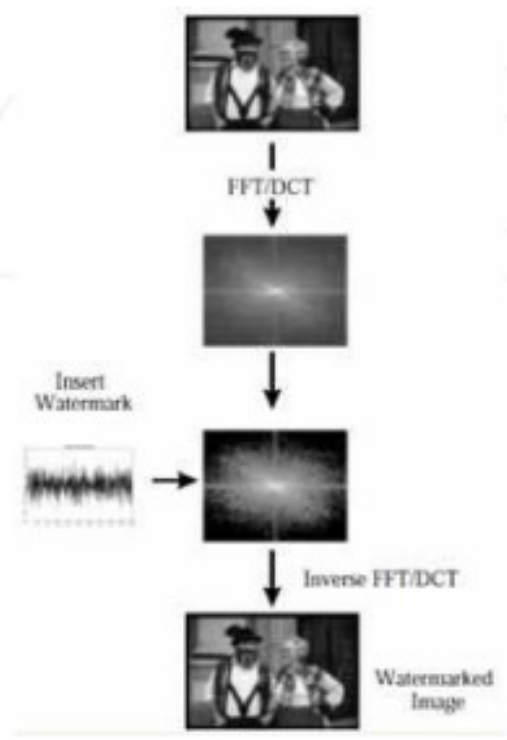

Fig 1 : Watermarking in Spectral Domain 


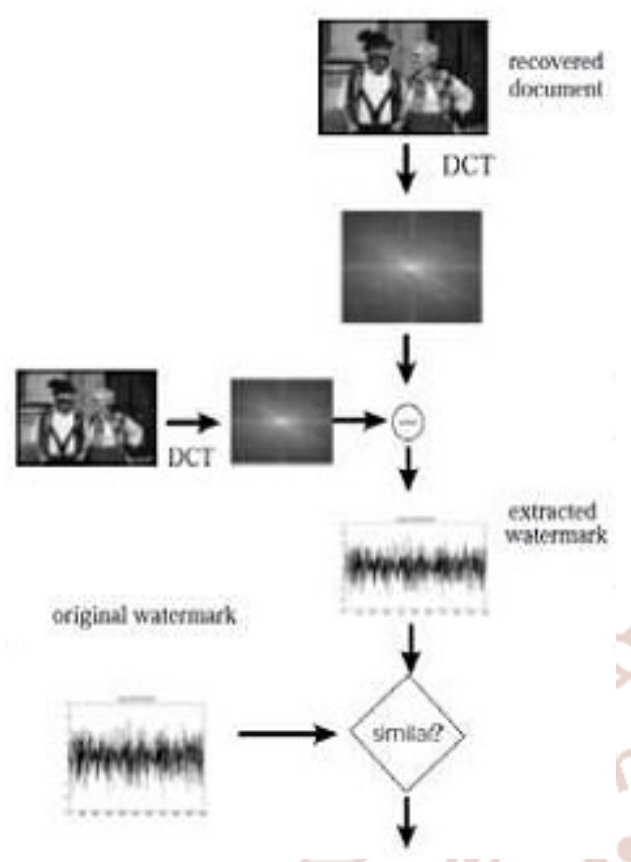

Fig 2: Watermark Extraction Process

\section{a. Proposed Embedding Algorithm}

(1) Information signal i.e. image is taken as input, which has to be hidden in audio.

(2) Convert it in to frequency domain by taking its Discrete Cosine Transform to decompose it into subbands.

(3) Result in transformed image.

(4) Similarly, take carrier audio signal convert it in to frequency domain by taking its Discrete Cosine Transform to decompose it into sub-bands.

(5) Form embedded signal by adding transformed information \& carrier signal to achieve watermarked sub-band, which will hide image sub-bands into audio sub-bands, it is called sub-band coding.

(6) Insert secret password key for additional security.

(7) The take Inverse Discrete Cosine Transform to reconstruct audio without changing its size i.e. convert it into time domain.

(8) Obtain watermarked audio \& plot amplitude spectrum, spectrogram for watermarked \& original audio.

\subsection{Proposed Extraction Algorithm}

In our proposed work we have used Transformation (DCT) technique for watermarking of image (Original Signal) into Audio (Carrier). Image to audio watermarking is challenging than an image to image or audio to audio watermarking technique due to wider dynamic range of the HAS in comparison with human visual system (HVS) [10]. By using Frequency masking and temporal masking the original signal can be hidden in the carrier signal. For security purpose a Password (Owner's Key) is also used in this work, which is needed at the time of embedding and for detection. A color image logo has been used as data and audio is taken as carrier.

Frequency (simultaneous) masking is a frequency domain observable fact where low levels signal (the maskee) can be made inaudible (masked) by a simultaneously appearing stronger signal (the masker) if the masker and maskee are close enough to each other in frequency [5]. A masking threshold can be found and is the level below which the audio signal is not audible. Thus, frequency domain is a good region to check for the possible areas that have imperceptibility. For embedding and extraction, the following steps are used:
(1) Select the watermarked audio which is containing data image hidden in it.

(2) Convert it in to frequency domain by taking its Discrete Cosine Transform, which will decompose the watermarked audio in sub-bands.

(3) Remove the of password from the Merged DCT Coefficients.

(4) Perform sub-band Demapping to obtain original data image.

(5) Extract image by subtracting watermarked image \&

(6) The take Inverse Discrete Cosine Transform of extracted sub-bands i.e. reconstruction of extracted data Image from subbands \& convert it into time domain.

(7) Calculate MSE, PSNR \& SSIM for Original vs Extracted Image for performance comparison.

\section{Simulation Results}

In this work an image logo is taken which is of size $284 \times 177$ as data object called original image, \& an audio of size $158 \mathrm{~KB}$ and duration of $0.7 \mathrm{Sec}$, is taken as carrier object. The proposed algorithm has been applied to hide the data object in carrier object. Then spectrum of the original image and Scalogram of carrier audio has been analyzed. audio signal. 


\subsection{Image to be Hidden}

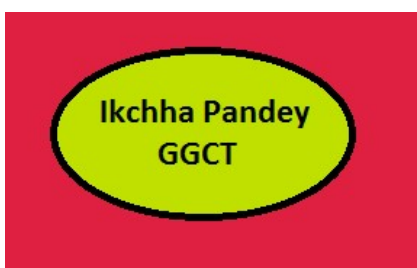

Fig 3: Original Image

\subsection{Original Image Spectrum}

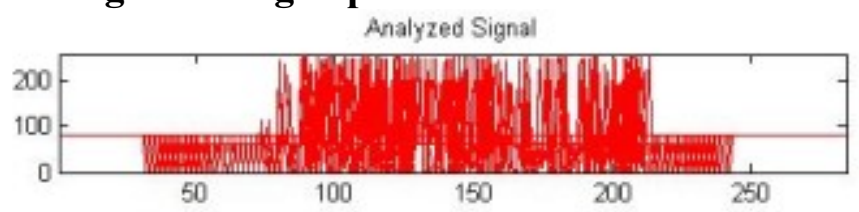

Fig 4: Spectrum of Original Image

\subsection{Original Image Scalogram}

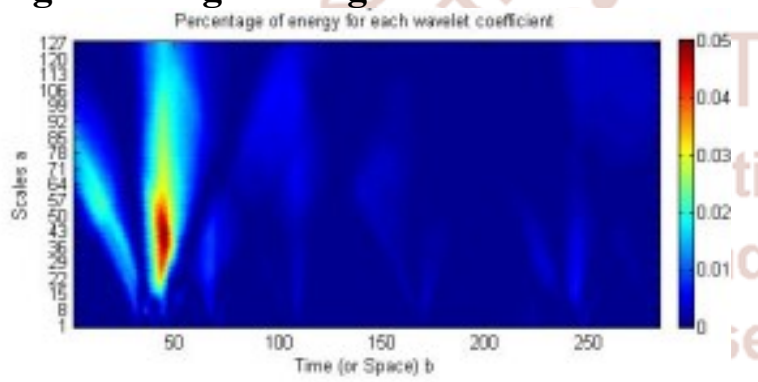

Fig 5: Original Image Scalogram

\subsection{Detected / Extracted Image}

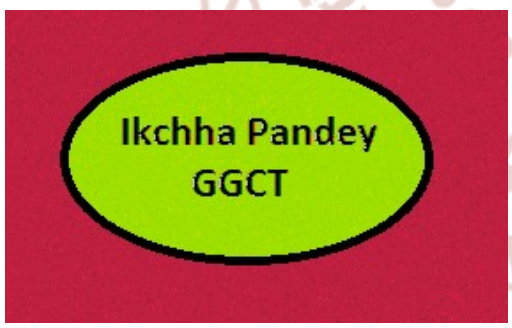

Fig 6: Detected Image from Watermarked Audio after Extraction

\subsection{Detected Image Spectrum}

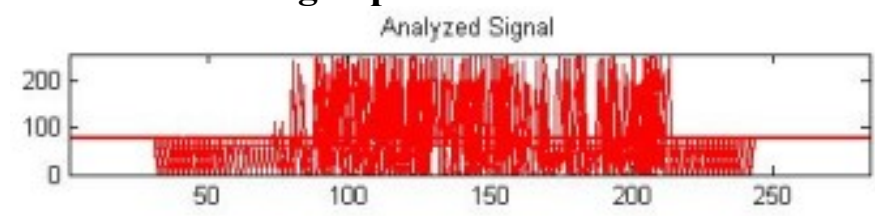

Fig 7: Spectrum of Detected Image

\subsection{Detected Image Scalogram}

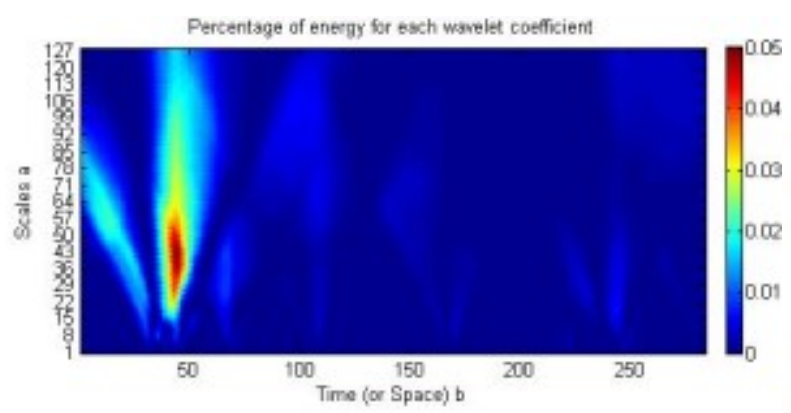

Fig 8: Detected Image Scalogram

5.7 Amplitude Spectrum of the Un-Watermarked Audio

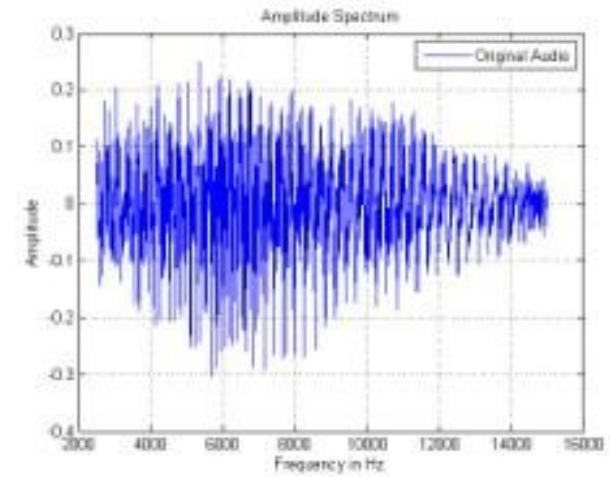

Figure 9: Unwatermarked Original Host Audio

5.8 Amplitude Spectrum of the Watermarked Audio

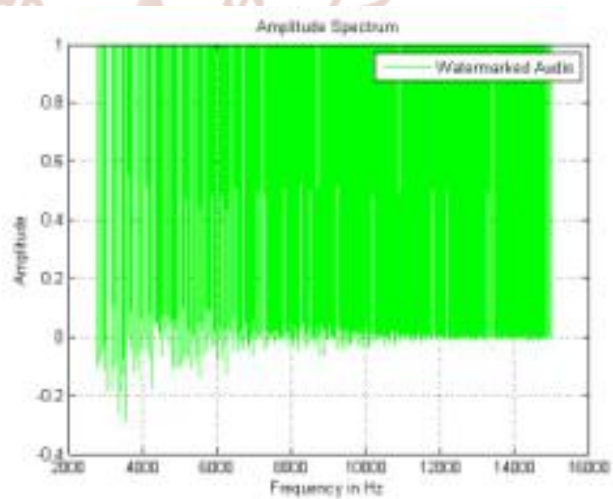

Fig 10: Watermarked Audio 


\subsection{Combined Amplitude Spectrum}

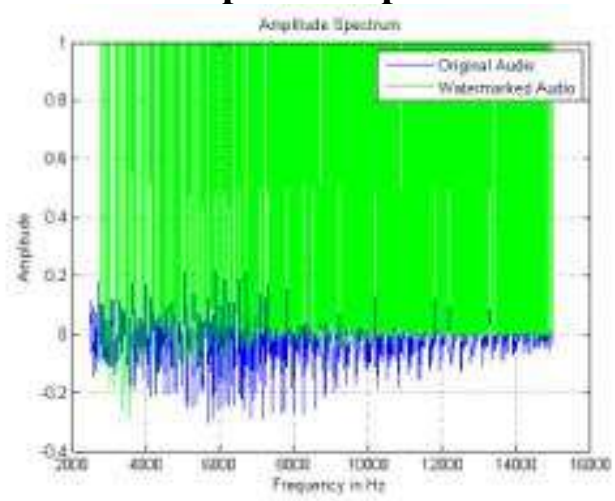

Fig 11: Original \& Watermarked Audio Spectrum

It has been clearly seen from the above figure that image is overlapped or mapped with positive values of audio components.

\subsection{Difference of the Amplitude Spectrum}

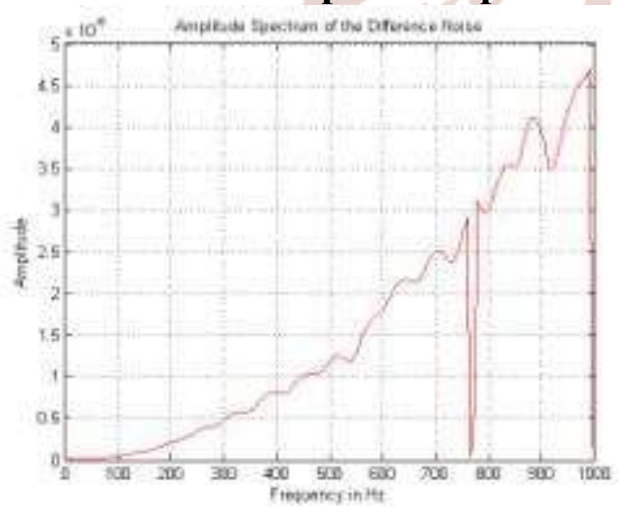

Fig 12: Original \& Watermarked Audio Spectrum Difference

\begin{tabular}{|c|c|c|c|c|c|c|}
\hline \multirow{2}{*}{ Imagm } & \multicolumn{3}{|c|}{ PSNR } & \multicolumn{3}{c|}{ MSE } \\
\cline { 2 - 7 } & LSE & DCT & DWT & LSE & DCT & DWT \\
\hline Jet [1] & 469015 & 47.9532 & 46.7300 & 05325 & 63320 & 13912 \\
\hline Boboon[1] & 473214 & 48.2992 & 46.9262 & 0.4534 & 0.3130 & 13610 \\
\hline This Work & & 49.42 & & & 0.75 & - \\
\hline
\end{tabular}

Table 1: PSNR \& MSE Results Comparison

\begin{tabular}{|c|c|c|}
\hline \multirow{2}{*}{ SNR } & Buse Paper [2] & This Work \\
\cline { 2 - 3 } & 2202 & 2357 \\
\hline
\end{tabular}

Table 2: SNR Results Comparison of Audio

\section{Conclusions}

In this work, a new watermarking based on Discrete Cosine Transform Sub-band coding scheme is presented. This method uses the time domain signal $\&$ process it in frequency domain, while time domain features of the carrier remains same, so no one can identify the hidden data into it. The algorithm is based on Psychoacoustic Auditory Model \& Spread Spectrum theory. It generates a watermark signal using spread spectrum theory \& embeds it into the signal by measuring the masking threshold. Since the watermark is shaped to lie below the masking threshold, the difference between the original \& the watermarked copy is imperceptible. Recovery of the watermark is performed without the knowledge of the original signal. In this work an image logo is taken which is of size $284 \times 177$ as data object called original image, \& an audio of size $158 \mathrm{~KB}$ and duration of $0.7 \mathrm{Sec}$, is taken as carrier object.

\subsection{Spectrogram (Time-Frequency) Analysis of References}

\section{Audio}
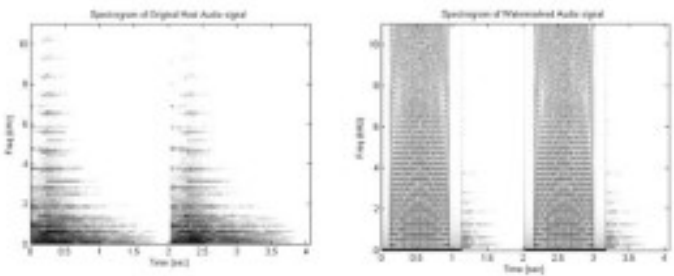

Fig 13: Spectrogram of Original \& Watermarked Audio

\subsection{Simulation Results Summary}

Image histogram similarity $=0.999999$

Image spatiogram similarity $=0.999998$

SSIM value $=0.9988$

M.S.E.: 1.02

PSNR: $42.4257214 \mathrm{~dB}$
1) S. Chandran et-al, "Performance Analysis of LSB, DCT, and DWT for Digital Watermarking Application using Steganography", IEEE International Conference on Electrical, Electronics, Signals, Communication and Optimization (EESCO) - 2015.

2) M. K. Pandey et-al, "Audio Watermarking by Spreading Echo in Time Domain Using Pseudo Noise Gray Sequence", IEEE International Conference on Industrial Instrumentation and Control (ICIC), India, May, 2015.

3) Neha Bansal et-al, "Comparative Analysis of LSB, DCT \& DWT for Digital Watermarking”, 2nd IEEE International Conference on 
Computing for Sustainable Global Development (INDIACom), Pp. 40 - 45, New Delhi, 2015.

4) Saeed S. et-al, "A Watermarking Method for Digital Speech Self-Recovery", IEEE/ACM Transactions on Audio, Speech, \& Language Processing, Volume 23, Number 11, November 2015.

5) Sha Wang et-al, "Adaptive Watermarking and Tree Structure Based Image Quality Estimation", IEEE Transactions on Multimedia, Volume 16, Number 2, February 2014.

6) Ms. Komal V. Goenka et-al, "Overview of Audio Watermarking Techniques", IJETAE, Volume 2, Issue 2, February 2012.

7) Shweta Sharma et-al, "Survey on Different Level of Audio Watermarking Techniques", International Journal of Computer Applications (IJCA), Volume 49-No.10, July 2012.

8) Md. Iqbal Hasan Sarker et-al, "FFT-Based Audio Watermarking Method with a Gray Image for Copyright Protection", International Journal of Advanced Science and Technology, Volume 47, October, 2012.

9) B.K. Singh et-al, "Digital Audio Watermarking: An Overview", International Journal of Electronics and Computer Science Engineering (IJECSE), Volume 4, Number 4, 2013.

10) Dhananjay Yadav et-al, "Reversible Data Hiding Techniques", International Journal of Electronics and Computer Science Engineering (IJECSE), Volume 1, Number 2, 2013.

11) Ali Al-Haj et-al, "DWT-Based Audio Watermarking", The International Arab Journal of Information Technology, Vol. 8, No. 3, July 2011.

12) N.F. Johnson, S. Jajodia, and Z. Duric, Information hiding: Steganography and watermarking attacks and countermeasures, Kluwer academic Publishers, 2000.

13) M. Goresky, A. M. Klapper, Fibonacci, and Galois, "Representations of FeedbackWithCarry Shift Registers," IEEE Transaction on Information Theory, Vol. 48, No. 11, pp. 28262836. 2002.
14) C. Shoemaker, "Hidden bits: A survey of techniques for digital watermarking," Independent study, EER 290, spring 2002.

15) B. Dumitrescu, and A. B. Rad, "A Method for Designing the Double-Density Dual-Tree Discrete Wavelet Transform," Proc. Int. TICSP Workshop on Local and Non-Local Approximation in Image Processing, Lausanne, Switzerland, Aug. 2008.

16) S. Katzenbeisser, and F.A.P. Petitcolas, Information hiding techniques for steganography and digital watermarking, Artech House Publishers, 2000.

17) G. Voyatzis, and I. Pitas, "Applications of toral automorphisms in image watermarking," Proceedings of International Conference on Image Processing, vol. 1, pp. 237-240, 1996.

18) S.A. Craver, B. Liu, and M. Wu, "What can we reasonably expect from watermarks? Applications of Signal Processing to Audio and Acoustics.”IEEE, pp. 223-226, 2001.

19) J. Bloom, I. Cox, T. Kalker, J. Linnartz, M. Miller, and C. Traw. "Copy protection for DVD video," Proceedings of the IEEE, vo. 7, Issue 87, pp. 1267- 1276, 1999.

20) F.A.P Petitcolas. "Watermarking schemes evaluation," IEEE Signal Processing Magazine, -Volume 17, Issue 5, pp.58-64, 2000.

21) M. Steinebach, F. Petitcolas, F. Raynal, J. Dittmann, C. Fontaine, S. Seibel, et al., "Stirmark benchmark: Audio watermarking attacks," Proceedings of the International Conference on Information Technology: Coding and Computing, pp. 49-54, 2001, Las Vegas, Nevada.

22) X. Wang, and H. Zhao, "A Blind Audio Watermarking Robust Against Synchronization Attacks," CIS 2005, Part II, LNAI 3802, pp. 617622, 2005.

23) E. Zwicker, and H. Fastl, Psychoacoustics. Springer Verlag, Berlin, Germany, 1999.

24) X. Wang , P. Niu, and H. Yang, "A robust digital audio watermarking based on statistics charecteristics," Elsevier Lt 